\title{
Examination of the Closely Linked Dominant Adaptive Gene Hypothesis as an Alternative to Single Gene Heterosis Associated with $l(2) 55 i$ in Drosophila melanogaster")
}

\author{
Terumi MukaI ${ }^{2)}$ and Allan B. Burdick \\ Department of Biological Sciences, \\ Purdue University, Lafayette, Indiana, U.S.A.
}

Received January 30, 1961

An apparent example of single gene heterosis associated with a recessive lethal gene, $l(2) 55 i$, in Drosophila melanogaster was given by Mukal and Burdick (1959), and the viability factors which contribute to the single gene heterosis were assessed by Schnick, Mukai and Burdick (1960).

MukaI and Burdick (1959) established four artificial lethal heterozygous populations, the constitutions of which were reported in the 1959 paper. In all these populations, even though the genetic backgrounds were different and the starting frequencies of lethal heterozygotes differed, the lethal reached almost the same equilibrium point which was about 0.42 of lethal heterozygotes.

When the frequencies of lethal heterozygotes were estimated in later generations, they showed a decrease. MukaI and Burdick (1960) proposed a hypothesis to explain this decrease which says that mutational "static" in the lethal-heterozygote-viability. modifying genetic background can reduce the frequency of a heterotic lethal gene even though the mutation-produced modifiers are not predominantly adverse for heterozygote viability.

Still another hypothesis for the behavior of this heterotic lethal must be considered and evaluated. The lethal gene might not be heterotic at all but rather might be closely linked to a dominant adaptive gene which would carry the lethal along, as it were, until recombination brought about a separation. The purpose of this report is to examine this dominant adaptive gene hypothesis and to assess its applicability to the case at hand. A rejection of this hypothesis would lend substantial strength to the previously mentioned interrelated hypotheses of single gene heterosis and mutational "static." In' this report a mathematical model based on the linked dominant adaptive gene hypothesis is developed, computations made by a digital computer are given, and the results are compared with the experimental data.

\section{Theoretical Considerations}

MuKaI and Burdick (1959) showed that the superviability of the lethal heterozygotes in question was due to better female fecundity and stronger mating ability of males

1) This work has been supported by research grant E-1428 from the National Institutes of Health.

2) Present address: National Institute of Genetics, Misima, Japan. 
in the early generations. Therefore, the mathematical model was based upon the above finding and parameters for mating ability and fecundity were included.

\section{Definition of parameters:}

$a=$ recessive lethal gene, the wild-type allele being $A$.

$D=$ dominant adaptive gene, the wild-type being $d$.

$f=$ relative female fecundity of $D$-carrying (homozygous and heterozygous) flies, that of $d d$ being 1 .

$p=$ recombination frequency between genes $A$ and $D$.

$\mathrm{Q}_{i}=$ frequency of lethal heterozygotes in the $i$-th generation. MuKaI and Burdick (1959) scored the initial population as Generation 1 , but in this report we call the initial population Generation 0.

We make the assumptions that the lethal gene is completely recessive, the dominant adaptive gene is completely dominant and produces its superviability effect in fecundity and mating ability, and that there are no position effects and no interactions between loci.

Mathematical model: Under the above assumptions, the zygotic array shown in Table 1 can be obtained in an arbitrary (the $i$-th) generation.

Table 1. Zygotic array in an arbitrary generation

\begin{tabular}{c|c|c|c|c}
\hline Genotype & $\begin{array}{c}\text { Female } \\
\text { fecundity }\end{array}$ & $\begin{array}{c}\text { Male mating } \\
\text { ability }\end{array}$ & $\begin{array}{c}\text { Relative } \\
\text { frequency }\end{array}$ & $\begin{array}{c}\text { Frequency in } \\
\text { next generation* }\end{array}$ \\
\hline$\frac{D A}{D A}$ & $f$ & $m$ & $\mathrm{t}_{1}^{(i)}$ & $\mathrm{q}_{1}^{(i+1)}$ \\
$\frac{D A}{d A}$ & $f$ & $m$ & $\mathrm{t}_{2}^{(i)}$ & $\mathrm{q}_{2}^{(i+1)}$ \\
$\frac{d A}{d A}$ & 1 & 1 & $\mathrm{t}_{3}^{(i)}$ & $\mathrm{q}_{8}^{(i+1)}$ \\
$\frac{D A}{D a}$ & $f$ & $m$ & $\mathrm{t}_{4}^{(i)}$ & $\mathrm{q}_{4}^{(i+1)}$ \\
$\frac{D A}{d a}$ & $f$ & $m$ & $\mathrm{t}_{5}^{(i)}$ & $\mathrm{q}_{5}^{(i+1)}$ \\
$\frac{d A}{D a}$ & $f$ & $m$ & $\mathrm{t}_{6}^{(i)}$ & $\mathrm{q}_{8}^{(i+1)}$ \\
$\frac{d A}{d a}$ & 1 & 1 & $\mathrm{t}_{7}^{(i)}$ & $\mathrm{q}_{7}^{(i+1)}$ \\
\hline
\end{tabular}

* This is not a relative frequency but a frequency prior to division by the total.

After one generation's mating, the frequency of each genotype $\left(\mathrm{q}^{(i+1)}, j=1,2, \ldots\right.$, 7) can be obtained as shown in Table 2.

The relative frequency of each genotype in the $(i+1)$ th generation can be expressed as 


$$
\mathrm{t}_{j}^{(i+1)}=-\frac{\mathrm{qj}^{(i+1)}}{\sum_{j} \mathrm{qj}^{(i+1)}} \quad \text { where } j=1,2, \cdots, 7 .
$$

Therefore, the frequency of lethal heterozygotes in the $(i+1)$ th generation $\left(Q_{t+1}\right)$ can be expressed as

$$
\mathrm{Q}_{i+1}=\sum_{j=4}^{7} \mathrm{t}_{j}^{(i+1)}
$$

Table 2. Recurrence formulae of the frequencies of lethal heterozygotes

$$
\begin{aligned}
& \mathrm{q}_{1}{ }^{(i+1)}=\frac{1}{4} m f\left(2 \mathrm{t}_{1}{ }^{(i)}+\mathrm{t}_{2}{ }^{(b)}+\mathrm{t}_{4}^{(i)}+\mathrm{t}_{5}^{(i)}\right)\left\{2 \mathrm{t}_{1}^{(i)}+\mathrm{t}_{2}{ }^{(i)}+\mathrm{t}_{4}{ }^{(i)}+\mathrm{t}_{5}^{(i)}-p\left(\mathrm{t}_{5}^{(i)}-\mathrm{t}_{8}{ }^{(i)}\right)\right\} \\
& \mathrm{q}_{\mathrm{s}}{ }^{(i+1)}=\frac{1}{4} m f\left\{\left(\mathrm{t}_{2}{ }^{(i)}+p t_{5}{ }^{(i)}+(1-p) \mathrm{t}_{8}{ }^{(i)}\right)\left(2 \mathrm{t}_{1}{ }^{(i)}+\mathrm{t}_{4}{ }^{(i)}+\mathrm{t}_{5}{ }^{(i)}\right)+\mathrm{t}_{2}{ }^{(i)}\left(2 \mathrm{t}_{2}{ }^{(i)}+2 \mathrm{t}_{2}{ }^{(i)}\right.\right. \\
& \left.\left.+t_{4}^{(i)}+t_{5}{ }^{(i)}+t_{6}^{(i)}\right)+t_{8}^{(i)}\left(2 t_{1}^{(i)}+t_{2}^{(i)}+t_{4}^{(i)}+(1-p) t_{5}^{(i)}+p t_{8}^{(i)}\right)\right\} \\
& +\frac{\left(2 \mathrm{t}_{3}(i)+\mathrm{t}_{7}^{(i)}\right)}{4}\left\{f\left(2 \mathrm{t}_{1}^{(i)}+\mathrm{t}_{2}^{(i)}+\mathrm{t}_{4}^{(i)}+(1-p) \mathrm{t}_{5}^{(i)}+p \mathrm{t}_{6}^{(i)}\right)\right. \\
& \left.+m\left(2 \mathrm{t}_{1}{ }^{(i)}+\mathrm{t}_{2}^{(i)}+\mathrm{t}^{(i)}+\mathrm{t}_{5}^{(i)}\right)\right\} \\
& \mathrm{q}_{3}{ }^{(i+1)}=\frac{1}{4}\left\{m\left(\mathrm{t}_{2}^{(i)}+\mathrm{t}_{8}{ }^{(i)}\right)+\left(2 \mathrm{t}_{3}{ }^{(i)}+\mathrm{t}_{7}{ }^{(i)}\right)\right\}\left[f\left\{\mathrm{t}_{2}^{\left({ }^{(i)}\right.}+p \mathrm{t}_{5}^{(i)}+(1-p) \mathrm{t}_{8}^{(i)}\right\}\right. \\
& \left.+\left(2 \mathrm{t}_{3}^{(i)}+\mathrm{t}_{7}^{(i)}\right)\right] \\
& \mathrm{q}_{\mathbf{6}^{(i+1)}}=\frac{m f}{4}\left[\left\{\mathrm{t}_{4}^{(i)}+p \mathrm{t}_{5}^{(i)}+(1-p) \mathrm{t}_{6}{ }^{(i)}\right\}\left(2 \mathrm{t}_{1}{ }^{(i)}+\mathrm{t}_{2}{ }^{(i)}+\mathrm{t}_{5}{ }^{(i)}\right)+\mathrm{t}_{4}{ }^{(i)}\left(2 \mathrm{t}_{1}{ }^{(i)}\right.\right. \\
& \left.\left.+\mathrm{t}_{3}{ }^{(i)}+2 \mathrm{t}_{6}{ }^{(i)}+\mathrm{t}_{5}^{(i)}+\mathrm{t}_{6}{ }^{(i)}\right)+\mathrm{t}_{8}{ }^{(i)}\left\{2 \mathrm{t}_{1}^{(i)}+\mathrm{t}_{2}{ }^{(i)}+\mathrm{t}_{4}^{(i)}+(1-p) \mathrm{t}_{\mathrm{s}}{ }^{(i)}+p \mathrm{t}_{\mathrm{\theta}}^{(i)}\right\}\right] \\
& \mathrm{q}_{\mathrm{s}}^{(i+1)}=\frac{m f}{4}\left[\left\{(1-p) \mathrm{t}_{5}^{(i)}+p \mathrm{t}_{6}^{(i)}\left(2 \mathrm{t}_{1}^{(i)}+\mathrm{t}_{2}^{(i)}+\mathrm{t}_{4}^{(i)}\right)\right\}\right. \\
& \left.+\mathrm{t}_{5}^{(i)}\left\{2 \mathrm{t}_{1}^{(i)}+\mathrm{t}_{2}^{(i)}+\mathrm{t}_{4}^{(i)}+2(1-p) \mathrm{t}_{5}^{(i)}+2 p \mathrm{t}_{6}^{(i)}\right\}\right] \\
& +\frac{\mathrm{t}_{7}^{(i)}}{4}\left[m\left(2 \mathrm{t}_{1}^{(i)}+\mathrm{t}_{2}^{(i)}+\mathrm{t}^{(i)}+\mathrm{t}_{5}^{(i)}\right)+f\left\{2 \mathrm{t}_{1}{ }^{(i)}+\mathrm{t}_{2}{ }^{(i)}+\mathrm{t}_{4}^{(i)}\right.\right. \\
& \left.\left.+(1-p) \mathbf{t}_{s}^{(i)}+p t_{B}^{(i)}\right\}\right]
\end{aligned}
$$

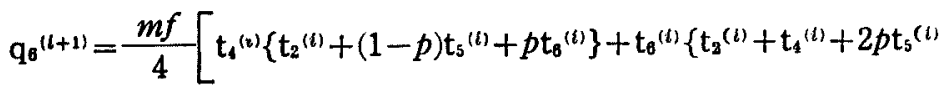

$$
\begin{aligned}
& \left.\left.+2(1-p) \mathrm{t}_{\mathrm{B}}^{(i)}\right\}+\mathrm{t}_{2}^{(i)}\left\{\mathrm{t}_{\mathrm{4}}^{(i)}+p \mathrm{t}_{\mathrm{s}^{(i)}}+(1-p) \mathrm{t}_{\mathrm{B}}{ }^{(i)}\right\}\right] \\
& \mathrm{q}_{7}{ }^{(l+1)}=\frac{m f}{4}\left[\mathrm{t}_{5}^{(i)}\left\{\mathrm{t}_{2}^{(i)}+p \mathrm{t}^{(i)}+(1-p) \mathrm{t}_{6}{ }^{(i)}\right\}+\left(\mathrm{t}_{\mathrm{3}}{ }^{(i)}+\mathrm{t}_{8}^{(i)}\right)\left\{(1-p) \mathrm{t}_{5}^{(i)}+p \mathrm{t}_{8}^{(i)}\right\}\right] \\
& +\frac{f}{4}\left[2 \mathrm{t}_{3}^{(l)}\left\{(1-p) \mathrm{t}_{5}^{(i)}+p \mathrm{t}_{8}^{(i)}\right\}+\mathrm{t}_{7}^{(i)}\left(\mathrm{t}_{2}^{(i)}+\mathrm{t}_{5}{ }^{(b)}+\mathrm{t}_{8}^{(i)}\right)\right]
\end{aligned}
$$

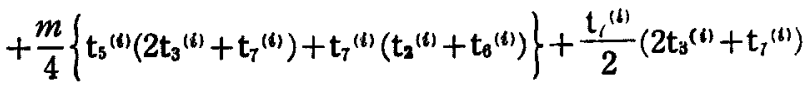




\section{Computation and Discusgion}

Computation was made by the digital computer in the statistical laboratory at Purdue University. Four cases with respect to mating ability of males and female fecundity were calculated. In every case, recombination values are assumed to be $0.00001 \sim 0.5, \mathrm{t}_{\mathrm{g}}{ }^{(0)}=1.00$, i. e., $\mathrm{Q}_{0}=1.00$.

Case 1. $m=1.44, f=1.70$ : If lethal heterozygotes show 1.44 times as much mating ability as wild-type males, and 1.70 times as much fecundity as wild-type females, the population of lethal heterozygotes reaches equilibrium at 0.42 frequency. Based upon the above findings, although they are not direct measurements but estimated values from the results of later generations (ScHNick, Mukal and Burdick 1960), we assume in this report that $D$-carrying homozygous and heterozygous individuals show $m=1.44$, and $f=1.70$, and that the lethal gene is completely recessive, having no effect on

Table 3. Expected frequency of lethal heterozygotes based upon $m=1.44, f=1.70$, and $\mathrm{Q}_{\mathrm{v}}=1(\mathrm{~A})$, and $m=2.34, f=1.00$, and $\mathrm{Q}_{0}=1(\mathrm{~B})$

\begin{tabular}{|c|c|c|c|c|c|c|c|c|}
\hline \multirow{3}{*}{ Generation } & \multicolumn{8}{|c|}{ Recombination value $(p)$} \\
\hline & \multicolumn{4}{|c|}{ A } & \multicolumn{4}{|c|}{ B } \\
\hline & $1 \times 10^{-5}$ & $1 \times 10^{-3}$ & $1 \times 10^{-1}$ & 0.50 & $1 \times 10^{-5}$ & $1 \times 10^{-3}$ & $1 \times 10^{-1}$ & 0.50 \\
\hline 1 & 0.667 & 0.667 & 0.667 & 0.667 & 0.667 & 0.667 & 0.667 & 0.667 \\
\hline 2 & 0.550 & 0.549 & 0.540 & 0.513 & 0.546 & 0.546 & 0.536 & 0.511 \\
\hline 3 & 0.494 & 0.494 & 0.470 & - & 0.490 & 0.489 & 0.466 & - \\
\hline 4 & 0.464 & 0.464 & 0.423 & - & 0.461 & 0.460 & - & - \\
\hline 5 & 0.447 & 0.446 & - & - & 0.444 & 0.443 & - & - \\
\hline 6 & 0.437 & 0.435 & - & - & 0.435 & 0.433 & - & - \\
\hline 7 & 0.430 & 0.429 & - & - & 0.429 & 0.427 & - & - \\
\hline 8 & 0.426 & 0.424 & - & - & 0.426 & 0.423 & - & - \\
\hline 9 & 0.424 & 0.420 & - & - & 0.423 & 0.420 & - & - \\
\hline 10 & 0.422 & 0.418 & 0.252 & 0.176 & 0.422 & 0.418 & 0.257 & 0.177 \\
\hline 11 & 0.421 & - & - & - & 0.421 & - & - & - \\
\hline 12 & 0.421 & - & - & - & 0.421 & - & - & - \\
\hline 13 & 0.420 & - & - & - & 0.421 & - & - & - \\
\hline 14 & - & - & - & - & 0.420 & - & - & - \\
\hline - & - & - & - & - & - & - & - & - \\
\hline 20 & 0.419 & 0.346 & 0.124 & 0.094 & 0.420 & 0.359 & 0.128 & 0.094 \\
\hline 30 & 0.398 & 0.182 & 0.078 & 0.064 & 0.406 & 0.200 & 0.080 & 0.064 \\
\hline 40 & 0.257 & 0.104 & 0.056 & 0.048 & 0.294 & 0.113 & 0.058 & 0.049 \\
\hline 50 & 0.134 & 0.071 & 0.044 & 0.039 & 0.155 & 0.075 & 0.045 & 0.039 \\
\hline 60 & 0.084 & 0.053 & 0.036 & 0.033 & 0.094 & 0.056 & 0.037 & 0.033 \\
\hline 70 & 0.061 & 0.042 & 0.031 & 0.028 & 0.066 & 0.044 & 0.031 & 0.028 \\
\hline 80 & 0.047 & 0.035 & 0.027 & 0.025 & 0.051 & 0.037 & 0.027 & 0.025 \\
\hline 90 & 0.038 & 0.030 & 0.023 & 0.022 & 0.041 & 0.031 & 0.024 & 0.022 \\
\hline 100 & 0.032 & 0.026 & 0.021 & 0.020 & 0.034 & 0.027 & 0.021 & 0.020 \\
\hline
\end{tabular}


heterozygote viability. The computational results are given in Table 3 .

Case 2. $m=2.34, f=1.00$ : If we attribute the forty-two per cent equilibrium frequency of lethal heterozygotes only to superior mating ability of lethal heterozygote males, the relative mating ability of these males should be 2.34 times as much as that of wild-type males (MukaI and Burdick 1959). For this reason, the second calculation was made, based upon $m=2.34$, and $f=1.00$. The results are presented in Table 3 .

Case 3. $m=1.00, f=2.34$ : If the forty-two per cent equilibrium frequency of lethal heterozygotes is attributed only to better female fecundity, the relative fecundity of lethal heterozygotes should be 2.34 times as much as that of wild-types just as in the case of male mating ability (Mukal and Burdick 1959). Therefore, the third calculation was made using $m=1$, and $f=2.34$. The results are presented in Table 4 .

Case 4. $m=1.00, f=1.21$ : Schnick, Mukal and Burdick (1960) directly measured the female fecundity and male mating ability of lethal heterozygotes in later genera-

Table 4. Expected frequency of lethal heterozygotes based upon $m=1, f=2.34$, and $\mathrm{Q}_{0}=1(\mathrm{~A})$, and $m=1, f=1.21$, and $\mathrm{Q}_{0}=1(\mathrm{~B})$

\begin{tabular}{|c|c|c|c|c|c|c|c|c|}
\hline \multirow{3}{*}{ Generation } & \multicolumn{8}{|c|}{ Recombination value $(p)$} \\
\hline & \multicolumn{4}{|c|}{ A } & \multicolumn{4}{|c|}{ B } \\
\hline & $1 \times 10^{-5}$ & $1 \times 10^{-3}$ & $1 \times 10^{-1}$ & 0.50 & $1 \times 10^{-5}$ & $1 \times 10^{-3}$ & $1 \times 10^{-1}$ & 0.50 \\
\hline 1 & 0.667 & 0.667 & 0.667 & 0.667 & 0.667 & 0.667 & 0.667 & 0.667 \\
\hline 2 & 0.546 & 0.545 & 0.536 & 0.511 & 0.512 & 0.512 & 0.508 & 0.503 \\
\hline 3 & 0.490 & 0.489 & 0.464 & - & 0.423 & 0.422 & - & - \\
\hline 4 & 0.460 & 0.460 & - & - & - & - & - & - \\
\hline 5 & 0.444 & 0.443 & - & - & - & - & - & - \\
\hline 6 & 0.435 & 0.433 & - & - & - & - & - & - \\
\hline 7 & 0.429 & 0.427 & - & - & - & - & - & - \\
\hline 8 & 0.426 & 0.423 & - & - & - & - & - & - \\
\hline 9 & 0.423 & - & - & - & - & - & - & - \\
\hline 10 & 0.422 & 0.417 & 0.245 & 0.174 & 0.230 & 0.230 & 0.195 & 0.170 \\
\hline 11 & 0.421 & - & - & - & - & - & - & - \\
\hline 12 & 0.421 & - & - & - & - & - & - & - \\
\hline 13 & 0.421 & - & - & - & - & - & - & - \\
\hline 14 & 0.420 & - & - & - & - & - & - & - \\
\hline- & - & - & - & - & - & - & - & - \\
\hline 20 & 0.419 & 0.350 & 0.122 & 0.093 & 0.179 & 0.178 & 0.114 & 0.092 \\
\hline 30 & 0.403 & 0.191 & 0.078 & 0.064 & 0.166 & 0.162 & 0.078 & 0.063 \\
\hline 40 & 0.283 & 0.109 & 0.056 & 0.048 & 0.161 & 0.153 & 0.058 & 0.048 \\
\hline 50 & 0.149 & 0.074 & 0.044 & 0.039 & 0.159 & 0.142 & 0.046 & 0.039 \\
\hline 60 & 0.092 & 0.055 & 0.036 & 0.033 & 0.158 & 0.126 & 0.037 & 0.032 \\
\hline 70 & 0.065 & 0.044 & 0.031 & 0.028 & 0.158 & 0.106 & 0.032 & 0.028 \\
\hline 80 & 0.050 & 0.036 & 0.027 & 0.025 & 0.158 & 0.085 & 0.027 & 0.025 \\
\hline 90 & 0.040 & 0.031 & 0.023 & 0.022 & 0.152 & 0.068 & 0.024 & 0.022 \\
\hline 100 & 0.034 & 0.027 & 0.021 & 0.020 & 0.144 & 0.055 & 0.022 & 0.020 \\
\hline
\end{tabular}


tions and detected that the relative fecundity of lethal heterozygotes was about 1.21 and that there were no differences in male mating ability, zygotic viability, or sperm competitive ability between the lethal heterozygotes and the wild-types at this time. Thus, $m=1.00$ and $f=1.21$ were assumed in the fourth calculation. The results are shown in Table 4.

Our findings relative to the above calculations can be summarized as follows:

The frequency of lethal heterozygotes in any given generation is an inverse function of $p$, the recombination distance between the lethal and the hypothetical dominant adaptive gene. (2) The tendency of linkage to maintain the lethal heterozygotes is clearly evident in the early generations of cases 1,2, and 3- the tighter the linkage, the more lethal heterozygotes. However, in later generations we see little difference between the various linkage intensities. For instance, in case 1 at generation 100 , the difference between $\left.p=0.00001: Q_{100}=0.032\right)$ and $p=0.50\left(Q_{100}=0.020\right)$ is quite small. (3) In case 4 , on the other hand, in the early generations all linkage values show a similar rapid decline of lethal frequencies, while in later generations the frequency values are quite dissimilar for different linkage intensities. And in addition, the frequency slopes

Table 5. Temporary equilibrium frequency of lethal heterozygotes and expected frequency based on the closely linked dominant adaptive gene hypothesis

\begin{tabular}{l|c|c|c}
\hline \hline & \multicolumn{3}{|c}{ Generation } \\
\hline Pop. 1 & $6-15$ & $45-49$ & $56-60$ \\
\hline Pop. 2 & - & $0.191 \pm 0.019$ \\
\hline & $0.420 \pm 0.019$ & - \\
\hline $0.424 \pm 0.017$ & $0.305 \pm 0.031$ & \\
\hline Calculation $^{*}$ & Generation \\
\hline $6-13$ & $40-50$ & $50-60$ \\
\hline
\end{tabular}

* Result of calculation based upon $m=1.44, f=1.70$ and $p=0.00001$.

are dissimilar through later generations $-p=0.00001$, for instance, shows almost no change in frequency between generations 50 and 100 while other linkage values produce a rapid decline during this period. (4) There appears to be a predictable period of generations during which the hypothetical dominant adaptive gene is favorable for keeping the lethal in the population and a later period during which it tends to drive the lethal out. The time when the change takes place is a function of linkage intensity; with tight linkage it is late, but with loose linkage, say 0.10 , the change comes before the 20th generation. And of course, the larger the assumed viability advantage of the dominant adaptive gene, the more dramatic is the shift of lethal heterozygote frequency. 
In the 1959 and 1960 papers by the present authors the temporary equilibrium frequencies of lethal heterozygotes in Population 1 (homozygous genetic background)

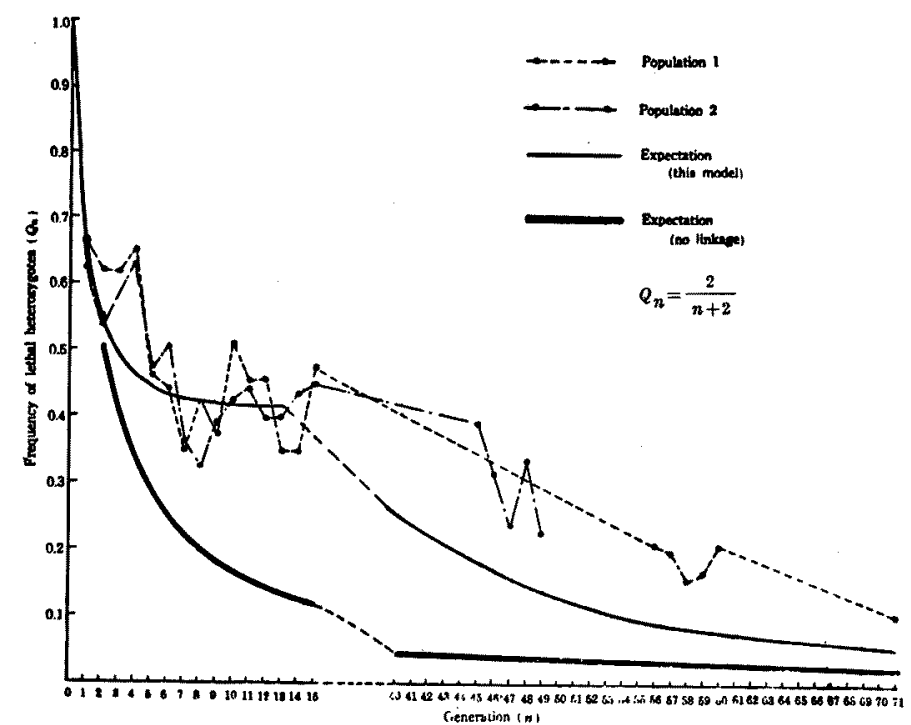

Fig. 1. Graphical presentation of the comparison between the observed data and the theoretical curve based upon the closely linked dominant adaptive gene hypothes:s $m=1.44, f=1.70$, and $p=0.00001$.

and Population 2 (heterozygous genetic background) were given. These results are summarized in Table 5 and graphically presented in Fig. 1 along with the frequency values that might have been expected under the most favorable conditions for the presently examined linked dominart adaptive gene hypothesis, namely, $m=1.44, f=1.70$, and $p=0.00001$ (from Table 3). The comparison indicates no reasonable basis for accepting the linked dominant gene hypothesis since, even with a $p$-value of 0.00001 , which must indeed be the minimum distance between two genes, the observed values are higher than the calculated.

None of these models appears to fit the actual data we have obtained for $l(2) 55 i$ populations. They all predict lower frequencies than those observed which leads us to reject the linked dominant adaptive gene hypothesis upon which the models are based. We feel that this strenghthens our previously proposed hypotheses that this $l(2) 55 i$ locus, in and of itself, is involved in an overdominance interaction, and that the slow, steady decline of lethal frequency that our populations have shown is attributable to mutational "static" in the lethal-heterozygote-viability-modifying genetic backgound, as described in MukaI and Burdick (1960). 


\section{Summary}

The second chromosome recessive lethal, $l(2) 5.5 i$, in Drosophila melanogaster is apparently involved in an extensively examined case of single gene heterosis. In the foregoing report we have tested and rejected the hypothesis that $1(2) 55 i$ might be closely linked to a dominant adaptive gene.

\section{Acknowledgement}

The authors would like to express their thanks to Dr. H. E. McKean and Mr. P. J. Cislack of the Purdue University Statistical Laboratory for help in developing the computer program for this study.

\section{Literature Cited}

Mukai, T. and A. B. Burdick 1959 Single gene heterosis associated with a second chromosome recessive lethal in Droscphila melanogaster. Genetics 44: 211-232. and 1960 Concerning equilibria of heterotic lethals in random mating popula. tions with particular reference to $l(2) 55 i$ in Drosophila melanogaster. Genetics 45: 15811594.

Schnick, S. M., T. Mukai and A. B. Burick 1960 Heterozygote viability of a second chromosome recessive lethal in Drosophila melanogaster. Genetics 45: 315-329. 